\title{
COVID-19 and nuclear cardiology: Introducing the "forward" virtual visit
}

\author{
George Angelidis ${ }^{1}$, Varvara Valotassiou ${ }^{1}$, Dimitrios Psimadas ${ }^{1}$, and Panagiotis Georgoulias ${ }^{1}$ \\ ${ }^{1}$ University of Thessaly
}

November 12, 2020

\section{COVID-19 and nuclear cardiology: Introducing the "forward" virtual visit}

Angelidis G, Valotassiou V, Psimadas D, Georgoulias P

Nuclear Medicine Laboratory, University of Thessaly, Larissa, Greece

We read with great interest the recent review article by Kaushik A, et al. concerning the potential role of digital health applications in the present pandemic situation [1]. As the authors noted, alternative tools are needed for the optimal management of cardiovascular patients, avoiding unnecessary visits to health care facilities. The severe acute respiratory syndrome - coronavirus - 2 (SARS-CoV-2) can invade the cardiovascular cells, potentially causing life-threatening cardiac impairment [2]. In particular, patients with pre-existing cardiovascular diseases are characterized by a higher risk of adverse cardiovascular events. Therefore, most of those referred for nuclear cardiology techniques are expected to be at higher risk of developing serious coronavirus disease 2019 (COVID-19) complications. However, the performance of the individually required diagnostic and follow-up procedures is important [3].

Telemedicine applications have been used in public health emergencies, leading to several advantages in terms of safety and efficacy. In the field of nuclear cardiology, the initial evaluation of patients' history and clinical features can take place remotely ("forward" virtual visit). This approach seems to be patientcentred (permitting an adequate case assessment) and conducive to self-quarantine (protecting patients, healthcare professionals, and the community from viral exposure). Importantly, possible clinical presentations of COVID-19 may be evaluated during the "forward" virtual visit, as well as information regarding travel and exposure histories. Moreover, local epidemiological information may be used to adjust screening pattern, and special measures could be developed (such as isolation in dedicated "hot" rooms) for patients with high-risk features. After the performance of the examination, telemedicine applications could be also used for the consultation with the patients.

Telemedicine applications may contribute to a better adjustment of nuclear cardiology services under the current demanding circumstances. Of course, no telemedicine programme can be created overnight, but this approach may be of value not only during the next months but also after the end of COVID-19 pandemic [4]. For example, our nuclear medicine laboratory is located in central Greece providing services to inhabitants of mountain villages, and nearby small islands. Consequently, the use of telemedicine applications could aid our practice in the future as well, particularly during the winter months when travelling by car or sea travels may be extremely demanding.

\section{REFERENCES}

1. Kaushik A, Patel S, Dubey K. Digital cardiovascular care in COVID-19 pandemic: A potential alternative? J Card Surg. 2020 Oct 10. 
2. Samidurai A, Das A. Cardiovascular complications associated with COVID-19 and potential therapeutic strategies. Int J Mol Sci. 2020;21(18):E6790.

3. Angelidis G, Valotassiou V, Psimadas D, Georgoulias P. A second wave of COVID-19 infections should not lead to a new disruption of services in nuclear cardiology. J Nucl Cardiol. 2020;1-2

4. Peretto G, De Luca G, Campochiaro C, et al. Telemedicine in myocarditis: Evolution of a mutidisciplinary "disease unit" at the time of COVID-19 pandemic. Am Heart J. 2020;229:121-126. 